\title{
Mercados Futuro e à Vista de Açúcar: uma análise empírica de eficiência versus arbitragem
}

\author{
Roseli da Silva ${ }^{1}$ \\ Rodrigo Takeuchi ${ }^{2,3}$
}

Resumo: Este trabalho testa a hipótese de eficiência relativa dos mercados futuro e à vista (spot) de açúcar, para dois horizontes de previsão, em contraposição à hipótese de arbitragem de commodities. Utiliza-se o modelo de não-arbitragem de Brenner e Kroner (1995) e aplica-se a metodologia de testes comparativos proposta por Kellard (2002), que usa a cointegração multivariada com restrição sobre o espaço de cointegração. A base de dados é formada a partir dos futuros do contrato número 11 negociado na Nybot (New York Board of Trade), dos preços no mercado à vista coletados pelo Cepea (Centro de Estudos Avançados em Economia Aplicada) e da taxa de juros doméstica, todas em bases diárias (mai/97 a dez/07). A correspondência da amostra é construída de acordo com os vencimentos dos contratos, considerando-se dois períodos de previsão: 28 e 56 dias. Em linhas gerais, as evidências empíricas encontradas suportam a adequação da metodologia de cointegração para análise de eficiência relativa nos mercados de açúcar, em contraposição à hipótese de arbitragem. Além disso, geram evidências fracas de ineficiência, resultados sujeitos à hipótese de estacionariedade do custo de carregamento, exceto pelo componente taxa de juros.

Palavras-chave: mercados futuros de açúcar, eficiência, arbitragem, cointegração.

1 Professora Doutora do Departamento de Economia da Faculdade de Economia, Administração e Contabilidade de Ribeirão Preto - FEA-RP/USP. Coordenadora do Observatório do Setor Sucroalcooleiro - FEA-RP/USP. E-mail: roselisilva@fearp.usp.br

2 Mestre em Economia Aplicada no Programa de Pós-Graduação em Economia PPGE -USP-RP. Bolsista do Observatório do Setor Sucroalcooleiro - FEA-RP/USP. E-mail: rtakeuchi@gmail.com

3 Os autores agradecem pelos comentários recebidos em apresentações de versões deste trabalho nos Seminários Internos do Observatório, no Seminário do Departamento de Economia FEA-RP/USP e no 8o․ Encontro Brasileiro de Finanças. Agradecemos, também, a assistência à pesquisa de Laís da Silva Ferreira. Erros e omissões são de responsabilidade dos autores. 


\begin{abstract}
This study tests the relative efficiency hypothesis of future and spot sugar markets for two forecast horizons, as opposed to commodity arbitrage. It was used a noarbitrage model of Brenner and Kroner (1995) and applied the methodology of comparative tests proposed by Kellard (2002), using multivariate cointegration methodology with restrictions on the cointegrated space. The database is formed by future prices of the number 11 contract traded on the Nybot (New York Board of Trade), domestic spot prices collected by Cepea (Center for Advanced Studies in Applied Economics) and the domestic interest rate, all on daily basis (May/97 to Dec/07). The matching sample is constructed by the futures contract maturity, considering two periods of forecast: 28 and 56 days. In general, the empirical evidences support the appropriateness of the cointegration methodology for analyzing the relative efficiency in the sugar markets, as opposed to arbitrage hypothesis, and generate weak evidence of inefficiency. Such results are subject to the assumption of stationarity of the cost of carry, except the interest rate component.
\end{abstract}

Key-words: sugar futures market, efficiency, arbitrage, cointegration.

Classificação JEL: G13, G14.

\title{
1. Introdução
}

Eficiência de mercado é um tema amplamente debatido nas áreas de finanças e economia, já que evidencia uma adequada assimilação de informações pelos agentes envolvidos e, consequentemente, uma precificação ótima dos ativos financeiros. Ao aumentar o número de participantes, tal eficiência propicia liquidez ao mercado.

O mercado de derivativos é o segmento de maior risco nas operações financeiras, sendo de fundamental importância a correta precificação de seus ativos para o gerenciamento de risco de empresas financeiras e não-financeiras. Esse mercado é composto pelos seguintes tipos de negociações: o contrato a termo, o contrato futuro, as opções e os swaps.

Neste trabalho, analisam-se os contratos futuros, que são acordos de compra e venda de um determinado ativo por certo preço, numa certa época no futuro, com padronizações de qualidade, quantidade, locais de entrega e datas específicas para liquidação dos negócios (HULL, 1997).

O foco de análise é a eficiência dos contratos futuros de açúcar, instrumento disponível às organizações agroindustriais do setor sucroalcooleiro para seu gerenciamento de risco. Ao fixar um preço de compra ou de venda de açúcar em uma data futura, reduz-se o risco de perdas decorrentes de variações nos preços do produto. Há que se considerar, entretanto, que a utilização de tal proteção contra variações nos preços (hedge) nunca é perfeita - depende da liquidez (volume de negociação) de tais contratos, além de requerer, das partes comprada e vendida, fundos disponíveis para as chamadas de margem ao longo do período de maturidade. 
Na data de vencimento de um contrato, o preço futuro deve convergir para o preço spot (à vista) - ocorrendo tal situação, diz-se que o mercado é eficiente. A verificação da hipótese de eficiência é necessária para que não haja a possibilidade de arbitragem, ou seja, a obtenção de lucro sem risco por meio da realização de negociações simultâneas em dois ou mais mercados. Se o preço futuro for maior que o preço spot na data de vencimento, um participante do mercado poderia comprar o ativo à vista e vendê-lo no mercado futuro, auferindo lucros sem risco, arbitrando.

O presente trabalho avalia, então, a hipótese de eficiência relativa dos mercados futuro e spot de açúcar, em contraposição à hipótese de arbitragem nos mesmos. Busca também averiguar se a metodologia de cointegração, amplamente aplicada para analisar a eficiência relativa entre mercados futuros e à vista, seria capaz de discernir entre eficiência e arbitragem no caso do mercado de açúcar.

Seguindo o modelo de arbitragem desenvolvido por Brenner e Kroner (1995) e a metodologia de testes comparativos propostos por Kellard (2002), aplicaram-se procedimentos econométricos robustos (cointegração multivariada e modelo de correção de erros) para avaliar a existência de cointegração entre preços à vista e futuros de açúcar, em conjunto com a taxa de juros doméstica.

A base de dados é formada a partir dos preços do contrato número 11 negociado na Nybot (New York Board of Trade) - a Bolsa de Mercadorias de Nova York, referência para hedgers e especuladores devido à maior liquidez em relação aos contratos negociados na BM\&F (Bolsa de Mercadorias e Futuros) -, das cotações no mercado à vista, calculadas pelo Cepea/Esalq (Centro de Estudos Avançados em Economia Aplicada, da Escola Superior de Agricultura Luiz de Queiroz), bem como da taxa de juros doméstica medida pela taxa Selic (Sistema Especial de Liquidação e Custódia), todas em bases diárias de 02 de maio de 1997 a 31 de dezembro de 2007. A correspondência da amostra foi estabelecida de acordo com os vencimentos dos contratos, considerando-se dois períodos de previsão: 28 e 56 dias, uma vez que metade dos contratos tem intervalo de um mês e a outra metade, de dois a cinco meses.

Os resultados encontrados revelam evidência fraca para a ineficiência, mas dão suporte à adequação da metodologia de cointegração para análise de eficiência relativa nos mercados de açúcar em contraposição à hipótese de arbitragem. Assume-se a hipótese de que o custo de carregamento seja estacionário, exceto pelo componente taxa de juros.

O artigo está dividido, além desta introdução, em mais cinco seções, contendo, na ordem: uma breve revisão teórica sobre mercados eficientes, com foco em commodities agrícolas; o modelo teórico e a metodologia econométrica aplicados no estudo; a metodologia de correspondência da amostra e a descrição dos dados utilizados, bem como os resultados dos testes para o mercado de açúcar no Brasil; e, por fim, as considerações finais. 
Mercados Futuro e à Vista de Açúcar:

uma análise empírica de eficiência versus arbitragem

\section{Hipótese de mercados eficientes - teoria e evidências}

A hipótese de eficiência de mercado tem origens bastante remotas, mas se consolidou na forma em que é debatida até hoje a partir do trabalho de Fama (1970). Conforme tal hipótese, mercados eficientes são aqueles em que os preços refletem completamente as informações disponíveis. São caracterizados por um grande número de pessoas bem informadas, cujas decisões de compra e venda exercem rápida influência sobre os preços.

Fama (1970) classifica a eficiência em três tipos: fraco, semiforte e forte. Sob eficiência fraca, supõe-se que todas as informações relevantes já estejam incorporadas no preço à vista e, portanto, os preços passados não seriam úteis para previsão. Essa forma de eficiência corresponde à hipótese de passeio aleatório para os ativos financeiros. Já a eficiência semiforte engloba, além das características do tipo fraco, a suposição de que todas as demais informações públicas também já estejam refletidas no preço à vista. Os preços à vista, sob eficiência forte, passariam a refletir, além dos conjuntos de informações anteriores, também informações privadas ou privilegiadas.

O objetivo do trabalho de Fama (1970) foi mostrar que nenhuma estratégia pode ser elaborada para retornos acima da média do mercado. Foram feitos testes para verificar se o mercado segue um passeio aleatório ${ }^{4}$, ou seja, se as variações dos preços não se correlacionam e nenhuma informação passada pode ser usada para prever futuros. O autor conclui que a hipótese de eficiência dos mercados é válida para o mercado acionário americano, indicando que o mesmo segue um passeio aleatório.

Malkiel (2003) argumenta que mercados financeiros eficientes são aqueles que não permitem aos investidores obterem retornos acima da média, à medida que esses agentes também não aceitam riscos acima da média. Para o autor, um investidor que escolhe aleatoriamente uma carteira de ativos no mercado de ações consegue o mesmo retorno que um investidor "profissional". O mercado pode ser eficiente mesmo que seus participantes sejam em parte irracionais. Nem mesmo em períodos de alta volatilidade dos preços, que não é explicada por fundamentos, seria possível obter retornos acima da média, segundo Malkiel (2003).

Como premissas básicas (PESARAN, 2005), a hipótese de eficiência de mercado assume: racionalidade dos investidores, no sentido de que são capazes de atualizar suas crenças à medida que novas informações estão disponíveis; arbitragem, ou seja, as decisões de investimento individuais satisfazem as condições de arbitragem; e racionalidade coletiva, em que os erros aleatórios de cada investidor se cancelam no mercado. Pesaran mostra que, se houver um número grande de participantes e nenhum deles dominar a formação de preços, pode ocorrer eficiência e não oportunidade de arbitragem. Adicionalmente, o autor aponta novas linhas de pesquisa que exploram limites à racionalidade dos agentes e à arbitragem, devido a requerimentos de liquidez e institucionais.

4 Um passeio aleatório é definido por $\gamma_{\mathrm{t}}=\gamma_{\mathrm{t}-1}+\varepsilon_{\mathrm{t}}$ sendo $\varepsilon_{\mathrm{t}}$ um ruído branco. 
A hipótese de eficiência já foi amplamente avaliada para o mercado acionário, principalmente considerando-se os testes de passeio aleatório. Muitos trabalhos também abordam o tema no mercado de commodities, e suas conclusões, em geral, divergem da que Fama encontrou para o mercado acionário americano.

Larson (1960), ao analisar a aleatoriedade das oscilações dos preços nos mercados futuros, argumentou que os atuantes no mercado de commodities tentam obter lucro antecipando o movimento dos preços. Para tal, utilizam novas informações que chegam ao mercado. Como o acontecimento dessas notícias é aleatório, as oscilações de preços também se dão de maneira aleatória. Porém, o autor faz uma ressalva:

No actual market behaves exactly like an ideal market. Many commodity futures markets approach the ideal, and differ principally in that traders react with varying skill to varying sources of information, and so some of the response to price-making forces is delayed. (LARSON, 1960, p. 316)

Stevenson e Bear (1970) analisaram os movimentos dos preços de milho e soja para detectar se o mercado seguia um passeio aleatório ou se existia uma tendência nas cotações dessas commodities que permitisse elaborar uma estratégia para obtenção de lucros. Na conclusão do trabalho, os autores encontram um movimento sistemático nos preços dos grãos - justamente o oposto de um movimento aleatório, que não oferece uma explicação satisfatória para as oscilações. Portanto, identificam uma tendência que possibilita lucro sob certos padrões de comercialização das commodities.

Cargill e Rausser (1975) utilizam filtros mecânicos para determinar se existe um comportamento sistemático dos preços futuros das commodities e a consequente possibilidade de obtenção de lucros. Os autores testam a hipótese de que incrementos nos preços futuros de um contrato são independentes e não correlacionados ao longo do tempo. A rejeição de tal hipótese é condição suficiente para se rejeitar a ocorrência de passeio aleatório, mostrando que o mercado não é eficiente. Foram examinadas sete commodities negociadas nas três principais bolsas americanas, aplicando-se os testes a 464 contratos. O objetivo foi assegurar um procedimento robusto, de modo a evitar que os resultados não fossem sensíveis apenas para determinado teste. Na conclusão, os autores afirmam,

It clearly appears that the random walk model must be rejected as a realistic description of commodity markets...the random walk does not serve as a reasonably accurate explanation of commodity market behavior. (CARGILL e RAUSSER, 1975, p. 1051)

Kellard et al. (1999) avaliam a hipótese de eficiência aplicando metodologia de cointegração e modelo de correção de erro, para um conjunto de mercados futuros de commodities e financeiros. Encontram resultados favoráveis à eficiência no longo prazo. Já no curto prazo, os autores evidenciam ineficiência, ao detectar 
mudanças no preço à vista devido a diferenças defasadas entre as cotações à vista e os futuros. Tal comportamento poderia ter sido evitado com a utilização de informações passadas para prever os movimentos do preço à vista.

McKenzie et al. (2002) estudam a eficiência dos mercados futuros de arroz nos Estados Unidos aplicando, igualmente, metodologia de cointegração e modelo de correção de erro. As previsões fora da amostra são comparadas às obtidas por modelos Arima a fim de avaliar o desempenho dos mercados futuros de açúcar como previsor. Os resultados também são favoráveis à hipótese de eficiência dos mercados futuros de arroz.

Os mercados futuros de soja e trigo foram avaliados por Wang (2003), da mesma forma, com instrumental de cointegração. Os resultados foram favoráveis à existência de eficiência nos mercados de soja. Por outro lado, para o mercado de trigo, apontou-se ineficiência, causada, provavelmente, por excesso de especulação e intervenção governamental.

Especificamente para o mercado brasileiro, Lima e Ohashi (1999) examinaram o comportamento da memória autorregressiva dos retornos dos preços futuros do açúcar entre janeiro de 1985 e junho de 1998, somando 3.318 observações. Foi estimado um modelo autorregressivo com filtro de Kalman, com coeficientes que variam no tempo. Pretendia-se mostrar que o aumento da eficiência de mercado estava relacionado à queda em sua memória autorregressiva. Segundo os autores, os preços não seguem um passeio aleatório, mas levantam a hipótese de que o mercado pode estar cada vez mais estável devido à menor variância dos coeficientes na metade final da amostra analisada. A interpretação é a de que tem aumentado o número de informações disponíveis, ao mesmo tempo em que os investidores as assimilam cada vez mais rapidamente. Na conclusão, Lima e Ohashi comentam: "Assim, para o investidor 'astuto', lucros extraordinários podem existir mesmo se o mercado financeiro estiver em bom funcionamento." (1999, p. 11).

Em síntese, o tema é bastante controverso, e os estudos não apontam um resultado conclusivo a respeito da eficiência de mercado, principalmente quando se tratam de commodities agrícolas.

Este trabalho, portanto, tem o objetivo de contribuir com a discussão, trabalhando com o mercado de açúcar a partir de uma metodologia econométrica diferente das utilizadas nos estudos citados.

\section{Modelo teórico e metodologia econométrica}

Mercados futuros e à vista relativamente eficientes requerem que o logaritmo dos preços futuros, $\mathrm{f}_{\mathrm{t}-\mathrm{j}}$, tomados i períodos antes da maturidade do contrato em $\mathrm{t}$, seja uma previsão não-viesada do logaritmo do preço à vista, $\mathrm{s}_{\mathrm{t}}$. Em outras palavras, se os mercados são eficientes, no sentido fraco, o lucro esperado de uma operação especulativa no mercado deve ser igual a zero. Sistematizando a relação entre o preço futuro e o à vista, tem-se, em logaritmos: 


$$
\mathrm{S}_{\mathrm{t}}=\mathrm{f}_{\mathrm{t}-1}+\varepsilon_{\mathrm{t}}
$$

Na qual o erro $\varepsilon_{t}$ tem o valor esperado zero da perspectiva do período $t$.

Se, como na equação 1, o preço futuro em t-1 é uma estimativa não-viesada do preço à vista em $t$, então se espera que haja alguma combinação linear entre esses preços que seja estacionária - ou seja, deve existir a e b tal que $z_{t}$ seja estacionária com média zero:

$$
\mathrm{Z}_{\mathrm{t}}=\mathrm{s}_{\mathrm{t}}-\mathrm{bf}_{\mathrm{t}-1}-\mathrm{a}
$$

Se, de fato, os dados confirmarem as hipóteses de que $b=1$ e que $z_{t}$ tem valor médio zero da perspectiva do tempo t, a hipótese de previsão não-viesada de longo prazo pode ser mantida, e os mercados à vista e futuro estarão em equilíbrio de longo prazo. Conclui-se, assim, que os mercados são eficientes em assimilar as informações disponíveis da melhor maneira possível. O parâmetro a não necessariamente precisa ser nulo, uma vez que podem existir custos de transação entre os mercados (CAMPBELL et al. 1997).

Se ambas as séries temporais de preços forem integradas de primeira ordem (I(1)), isto é, apresentarem uma raiz unitária, pode existir uma relação de longo prazo entre elas, expressa pelo vetor de cointegração, o que significa, nesse caso, a primeira etapa na avaliação da eficiência de mercado (condição necessária, mas não suficiente). Como condição suficiente, um teste de restrição de parâmetros deverá ser realizado.

Entretanto, Kellard (2002) questiona a adequação da metodologia de cointegração para avaliar a hipótese de eficiência de mercado, resgatando o trabalho teórico de Brenner e Kroner (1995), referenciado como modelo BK daqui em diante. Os autores constroem um modelo para o mercado de commodity que não requer hipóteses comportamentais para os participantes. Seguindo Kellard (2002), que produz uma versão discreta do modelo BK, apresenta-se o seguinte argumento:

Suponha que o logaritmo do preço à vista seja um passeio aleatório com drift $(\mu)$ :

$$
\Delta \mathrm{s}_{\mathrm{t}}=\mu+\mathrm{u}_{\mathrm{t}}
$$

Sendo $\mathrm{u}_{\mathrm{t}}$ estacionário e invertível.

Os preços futuros são formados por uma relação de não-arbitragem entre os contratos futuro e à vista, de tal forma que os investidores sejam indiferentes entre: (a) comprar a commodity à vista e mantê-la, arcando com custos de estocagem e retornos adequados; e (b) investir no ativo livre de risco e comprar a commodity mais tarde aos preços futuros cotados no momento presente. Essa é a relação de não-arbitragem, expressa por:

$$
F_{t}=S_{t} e^{D t}
$$


Sendo $D_{t}$ a soma da taxa de juros doméstica, $R_{t}$ do custo de estocagem, $W_{t}$, expresso como proporção do preço à vista e subtraindo o retorno conveniente $\mathrm{Y}_{\mathrm{t}}$ :

$$
D_{t}=R_{t}+W_{t}-Y_{t}
$$

Em seguida, substitui-se a versão logarítmica defasada em um período da equação 4 na equação 3 , resultando em:

$$
\mathrm{s}_{\mathrm{t}}-\mathrm{f}_{\mathrm{t}-1}=\mu-\mathrm{D}_{\mathrm{t}-1}+\mathrm{u}_{\mathrm{t}}
$$

A partir da equação 6, a relação correta a se observar no mercado de commodities não é a hipótese de previsor não-viesado dada pela equação 1 , mas, sim, esta última aumentada pela taxa de juros doméstica, pelos custos de estocagem e pelo retorno adequado. Considerando que custos de estocagem e retorno adequado possam ser estacionários, se o preço à vista, o futuro e a taxa de juros doméstica forem não-estacionárias, a condição necessária para eficiência passa a ser a existência de cointegração no sistema de três variáveis:

$$
s_{t}=\beta_{0}+\beta_{1} f_{t-1}+\beta_{2} R_{t-1}+v_{t}
$$

Em que $\mathrm{v}_{\mathrm{t}}=\left(\mathrm{W}_{\mathrm{t}-1}-\mathrm{Y}_{\mathrm{t}-1}\right)+\mathrm{u}_{\mathrm{t}}$, por hipótese, estacionário.

Se a formulação de BK pode ser considerada um modelo adequado para o mercado de commodities, há duas implicações sobre os resultados empíricos, que dependem basicamente do processo estocástico da taxa de juros doméstica. Se esta for estacionária, espera-se que haja cointegração entre o logaritmo dos preços à vista e futuro, com vetor [1,-1], o que reflete a condição de não-arbitragem e, portanto, de eficiência. Caso a taxa de juros doméstica apresente uma tendência estocástica, deve-se esperar um vetor de cointegração [1, -1, 1]. A não-rejeição dessa hipótese implica rejeição da eficiência de mercado no longo prazo, já que, se três variáveis são cointegradas entre si, elas não podem cointegrar duas a duas.

Para avaliar as condições de estacionariedade das séries temporais em questão, levando em conta os problemas de baixo poder e distorção de tamanho dos tradicionais testes Augmented Dickey-Fuller (ADF), Phillips-Perron (PP) e Kwiatkowski, Phillips, Schmidt e Shin (KPSS), amplamente apontados na literatura ${ }^{5}$, serão aplicados testes mais robustos.

$\mathrm{O}$ primeiro, desenvolvido a partir da linhagem $\mathrm{ADF}$, e que permite um estudo adequado da presença de componentes deterministas, será o teste ERS ou DF-GLS (ELLIOTT, ROTHENBERG e STOCK, 1996), que propõe uma modificação simples ao aplicar o ADF à série temporal previamente filtrada de seus componentes deterministas.

5 Veja, por exemplo, De Jong et al. (1992), Cochrane (1991) e Maddala e Kim (2003) este último apresenta uma boa revisão da literatura. 
O segundo teste, proposto por NG e Perron (1996 e 2001), segue a metodologia não-paramétrica dos testes PP, em que a matriz de variância dos estimadores dos parâmetros da equação de teste é consistente com heterocedasticidade e autocorrelação. Os autores propõem tratar os problemas associados aos testes usuais construindo estatísticas de testes para a série sem os componentes deterministas (estimados com uso de GLS, como no caso anterior).

Sendo as $N$ variáveis em $X$ integradas de primeira ordem (I(1)), ou seja, variáveis com uma tendência estocástica, pode existir uma relação de longo prazo entre elas, que será expressa por até (N-1) vetores de cointegração. Para tal análise, parte-se de um Vetor Autorregressivo (VAR) de ordem k:

$$
X_{t}=\sum_{i=1}^{k} A_{i} X_{t-i}+\mu_{0}+\mu_{1} t+\varepsilon_{t}
$$

Em que $\mu_{0}$ e $\mu_{1}$ são os vetores com parâmetros linear e angular da tendência determinista, $(\mathrm{t}), \mathrm{A}_{\mathrm{i}}, \mathrm{i}=1, \ldots, \mathrm{k}$ são as matrizes de coeficientes e $\varepsilon_{\mathrm{t}} \sim \operatorname{Niid}(0, \Sigma)$, com $\Sigma$ expressando a matriz de variância-covariância, isto é, o vetor de resíduos do $\operatorname{VAR}\left(\varepsilon_{\mathrm{t}}\right)$ deve ser independente e normalmente distribuído. Porém, para a validade assintótica das distribuições, basta que os resíduos sejam ruídos brancos (médias nulas, variâncias constantes e não-autocorrelacionados - ou seja, $\left.\Sigma=\sigma^{2} \mathrm{I}\right)$. É possível, conforme a equação 8, modelar componentes deterministas em conjunto. A adequada especificação do VAR é, então, a segunda etapa do trabalho empírico (a primeira é a verificação da ordem de integração), que utiliza critérios de informação para a seleção da defasagem adequada e testes específicos para a busca de componentes deterministas.

Na presença de séries temporais com raiz unitária (tendência estocástica), o VAR pode ser adequadamente reparametrizado e representado por:

$$
\Delta \mathrm{X}_{\mathrm{t}}=\delta_{0}+\delta_{1} \mathrm{t}+\Pi_{\mathrm{k}} \mathrm{X}_{\mathrm{t}-1}+\sum_{\mathrm{i}=0}^{\mathrm{k}-1} \Pi_{\mathrm{i}} \Delta \mathrm{X}_{\mathrm{t}-1}+\varepsilon_{\mathrm{t}}
$$

em que $\Delta$ representa a primeira diferença do vetor de variáveis $X ; \delta_{0}$ e $\delta_{1}$ são os vetores de constante e o coeficiente angular da tendência determinista $(t)$, que devem ser reinterpretados em relação à equação 1. A presença da constante expressa a tendência linear determinista para as séries em nível, e o coeficiente angular representa a tendência quadrática também para as séries em nível, o que generaliza as possibilidades de tendência determinista no modelo empírico; o vetor de resíduos $\left(\varepsilon_{\mathrm{t}}\right)$ do $\operatorname{VAR}$ é $\varepsilon_{\mathrm{t}} \sim \operatorname{Niid}(0, \Sigma), \operatorname{com} \Sigma=\sigma^{2} \mathrm{I}$.

A análise de cointegração na metodologia proposta por Johansen (1991) é, então, realizada por meio de testes sobre o posto da matriz de coeficientes das variáveis em nível defasadas de 1 período, $\prod_{k}$, que representa as propriedades de longo prazo do sistema, enquanto $\prod_{\mathrm{i}}$, $\mathrm{i}=1, \ldots, \mathrm{k}-1$ correspondem ao comportamento dinâmico de curto prazo. Se o posto da matriz $\prod_{k}$ é nulo, o sistema é não-estacionário - porém, sem qualquer relação de longo prazo identificável -, e um modelo econométrico deve ser construído para as primeiras 
diferenças das variáveis; se o posto é pleno (nesse caso, N-1), o sistema em nível já é estacionário; se o posto é reduzido (menor que N-1), há relações de longo prazo (vetores de cointegração) que tornam o sistema estacionário.

Para identificar a existência e o número de vetores de cointegração, há duas possibilidades: aplicar o teste do traço ou o do autovalor máximo. Neste trabalho, opta-se pela estatística do traço, mais robusta na ausência de normalidade:

$$
\operatorname{traço}=-\mathrm{T} \sum_{\mathrm{i}=\mathrm{r}+1}^{\mathrm{N}} \ln \left(1-\hat{\lambda}_{\mathrm{i}}\right)
$$

em que T é o tamanho da amostra; r, o número de vetores de cointegração distintos; e $\lambda_{\mathrm{i}}, \ldots \lambda_{\mathrm{N}}$, as $\mathrm{N}$ correlações canônicas ao quadrado entre $\mathrm{X}_{\mathrm{t}-\mathrm{p}}$ e $\Delta \mathrm{X}_{\mathrm{t}}$, em ordem descendente. Se o valor computado da estatística de traço é menor que o valor crítico, não se rejeita a hipótese de r vetores de cointegração (JUSELIUS, 2006).

A abordagem econométrica para inferir sobre a eficiência nos mercados de açúcar adotada neste trabalho consiste, inicialmente, em testar a existência de raiz unitária nas séries de preços e taxa doméstica de juros. Em seguida, avaliase a presença de cointegração entre elas, e por fim, testa-se a restrição sobre os parâmetros do vetor de cointegração.

\section{Eficiência versus arbitragem - evidências empíricas}

\subsection{Dados e correspondência da amostra}

Os dados utilizados neste estudo correspondem aos preços em dólar do contrato de açúcar número 11 negociado na Nybot durante o período de 2 de maio de 1997 a 31 de dezembro de 2007. O contrato tem quatro meses de vencimentos durante o ano (março, maio, julho e outubro) e foi escolhido dada a sua maior liquidez quando comparado ao contrato de açúcar da BM\&F. Pode ser negociado até o último dia útil do mês precedente ao mês de entrega da mercadoria.

Para o mercado spot, foram utilizados os preços, em dólares, divulgados pelo Cepea - as cotações são uma ponderação dos preços coletados junto a compradores, unidades produtoras (usinas) e grandes intermediários. A região de referência é o estado de São Paulo, que é subdividido em quatro regiões: Ribeirão Preto, Jaú, Assis e Piracicaba. O preço spot inclui dois impostos (ICMS e PIS/Cofins) e é apresentado em séries diárias para o mesmo período anteriormente citado.

Quaisquer diferenças de qualidade - já que o contrato $n .11$ negocia açúcar demerara (bruto) e o mercado à vista, o cristal -, bem como as relativas a custo de estocagem - o primeiro é para entrega no porto do exportador e o segundo, para retirada na usina -, supõe-se que as mesmas sejam estacionárias e estejam expressas em $v_{t}$ na equação 7 , ou que sejam captadas por uma constante não nula no vetor de cointegração.

A partir das bases disponíveis, elabora-se um banco de dados compatível com a maturidade dos contratos, que corresponda ao período de safra do açúcar, 
não permitindo sobreposição informacional que possa acarretar problemas de autocorrelação. Para tanto, o logaritmo dos preços à vista (spot) foi tomado no dia de vencimento dos futuros (últimos dias úteis de fevereiro, abril, junho e setembro - meses que antecedem os vencimentos), e os correspondentes preços futuros foram obtidos no período anterior, não ultrapassando dois meses.

Seguindo Kellard et al. (1999), os logaritmos dos preços futuros correspondentes foram selecionados com antecedência de 28 e 56 dias, a partir da maturidade de cada contrato, denominados, respectivamente, FUT1 e FUT2. Assim, foi possível a análise para dois horizontes de previsão, mantendo a regularidade que as séries temporais requerem. A escolha desses intervalos reflete também o fato de os contratos com último dia de negociação em abril e junho não terem, no período de 28 dias, sobreposição informacional com os contratos imediatamente anteriores - da mesma forma, os de fevereiro e setembro não o são no período de 56 dias. A mesma divisão deve ser mantida para a taxa de juros relevante, a taxa Selic, diária, capitalizada para 28 dias, IR1, e para 56 a partir da maturidade, IR2.

Como os futuros compatíveis com o preço à vista estão distantes do vencimento por uma fração da unidade de observação, denominada $\tau$, a equação 7 é adaptada:

$$
\mathrm{s}_{\mathrm{t}}=\beta_{0}+\beta_{1} \mathrm{f}_{\mathrm{t}-\tau}+\beta_{2} \mathrm{R}_{\mathrm{t}-\tau}+\mathrm{v}_{\mathrm{t}}
$$

Dessa forma, no ano, há quatro observações para cada série temporal, totalizando 42 observações no período de disponibilidade de dados para cada série.

Figura 1. Preços nos mercados futuro e à vista: horizonte de previsão de 28 dias (FUT1) e de 56 dias (FUT2).

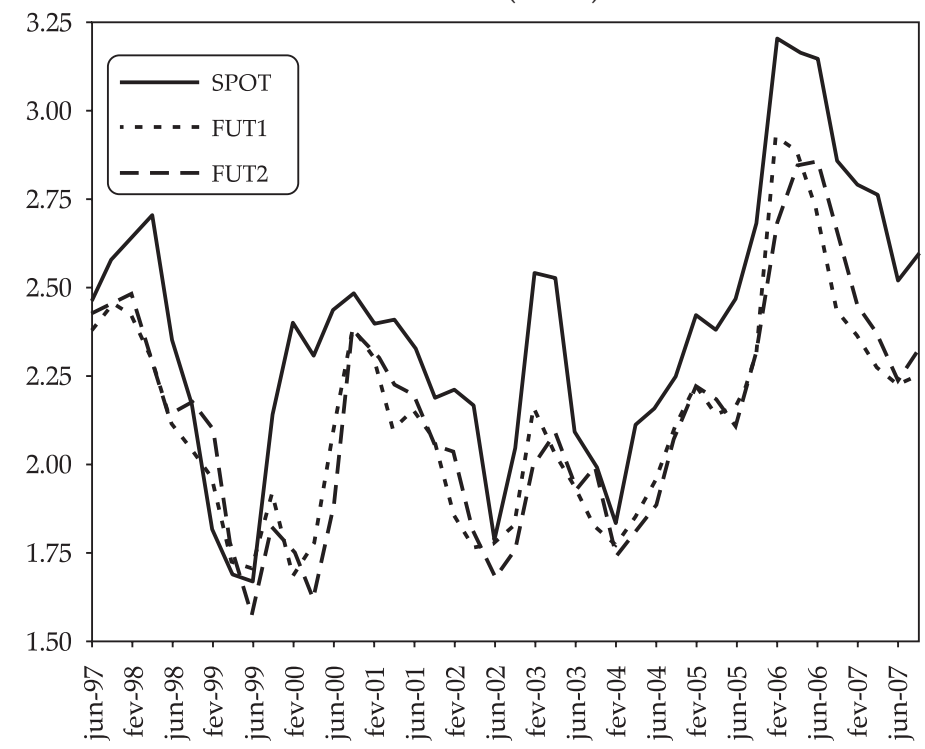

Fonte: Elaboração própria a partir de dados do Cepea e BM\&F. 
A observação do comportamento das séries sugere alguma fonte de nãoestacionariedade, embora seus respectivos correlogramas registrem decaimento exponencial com autocorrelação de primeira ordem acima de 0,81 para as três séries e significância individual até terceira defasagem - o que é bastante razoável, uma vez que as séries só apresentam quatro observações anuais e que, entre cada ano, há quatro meses sem vencimento de contrato.

Figura 2. Taxas de juros: horizonte de previsão de 28 e 56 dias.

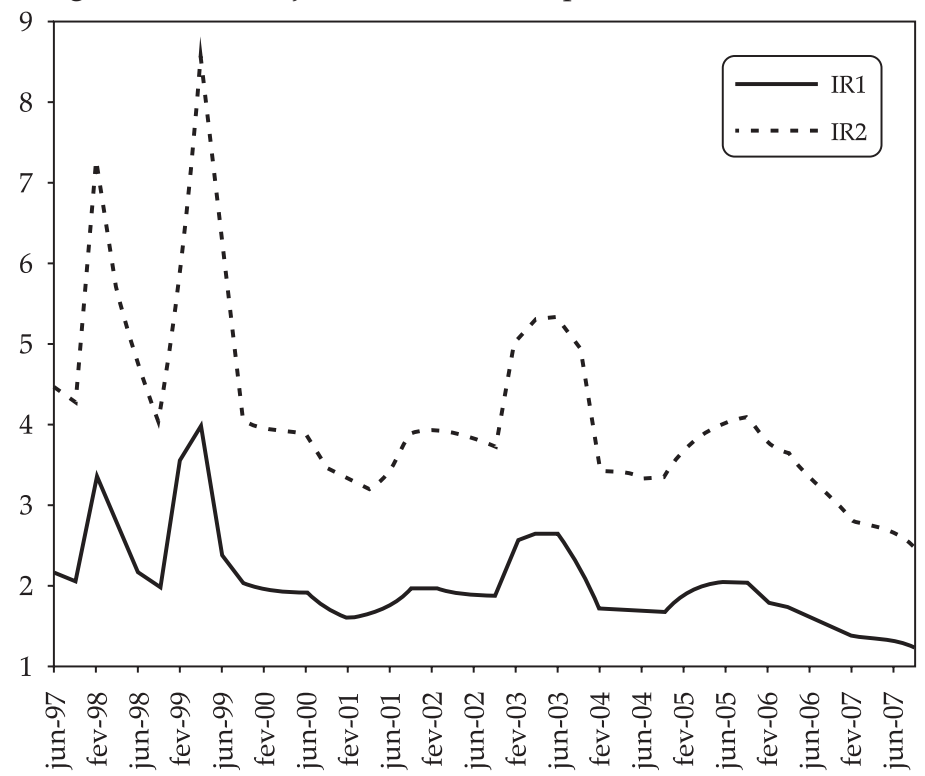

Fonte: Elaboração própria a partir de dados do Banco Central.

Já o comportamento das taxas de juros aponta dois picos no início da amostra, um em janeiro de 1998 e outro em março de 1999. Esses períodos expressam as crises internacionais e brasileira, além de um movimento de alta já no regime de metas de inflação, em meados de 2003. Exceto por esses picos, as séries parecem mais autorregressivas do que não-estacionárias, o mesmo indicado pelos correlogramas - tais outliers podem afetar tanto os testes univariados para raiz unitária quanto os de cointegração multivariada.

A Tabela 1 resume as estatísticas descritivas para os preços nos dois mercados e para as taxas de juros, para ambos os períodos de previsão, mostrando que o mercado à vista tem médias e medianas históricas mais elevadas, além de maior amplitude de variação e volalitidade, $50 \%$ superior ao mercado futuro. 
Tabela 1. Estatísticas descritivas das séries temporais.

\begin{tabular}{lccccc}
\hline & Vista & Futuro 1 & Futuro 2 & IR1 & IR2 \\
\hline Média & 11,55 & 8,80 & 8,90 & 2,10 & 4,14 \\
Mediana & 11,03 & 8,23 & 8,36 & 1,90 & 3,89 \\
Máximo & 24,67 & 18,61 & 17,48 & 3,97 & 8,59 \\
Mínimo & 5,29 & 5,39 & 4,82 & 1,21 & 2,43 \\
Desvio Padrão & 4,55 & 3,01 & 3,04 & 0,57 & 1,23 \\
\hline
\end{tabular}

Obs.: Nesta tabela, exclusivamente, os preços não estão em logaritmos.

Fonte: Elaboração própria a partir de dados do Banco Central.

\subsection{Cointegração, eficiência relativa e arbitragem}

Inicialmente, avalia-se a presença de não-estacionariedade nas séries por meio dos testes de raiz unitária. Esse método permite conhecer melhor o processo gerador dos dados individualmente, sendo valioso como informação adicional para a especificação do VAR necessário para a análise de cointegração. Além disso, mostra se cada mercado pode ser caracterizado como um passeio aleatório, indicando a possibilidade de eficiência absoluta em conjunto com algum modelo de equilíbrio para a formação de preços.

Tabela 2. Testes para raiz unitária para preços à vista, futuros e taxas de juros.

\begin{tabular}{lcc}
\hline & DF-GLS & NP \\
\hline Preço á vista (SPOT)* $^{*}$ & $-2,198$ & $-7,541$ \\
Preço Futuro (FUT1) & $-2,051$ & $-6,247$ \\
Preço Futuro (FUT2)* & $-1,989$ & $-6,089$ \\
Taxa de Juros (IR1)** & $-2,136$ & $-8,672$ \\
Taxa de Juros (IR2)* & $-3,595$ & $-14,965$ \\
\hline
\end{tabular}

Especificações: * Constante, tendência determinista e nenhuma defasagem (Seleção MAIC); ** Constante, tendência determinista e três defasagens (seleção MAIC).

Valores críticos a 5\%: -3,190 para DF-GLS e -17,3 para NP

Valores críticos a 10\%: $-2,890$ para DF-GLS e -12,2 para NP

Fonte: Elaboração própria.

Os testes foram realizados a partir de quatro defasagens, com identificação da defasagem ótima por meio do Critério de Informação de Akaike Modificado (Maic, na sigla em inglês). Foi significante a presença de constante e de tendência determinista, esta última avaliada por meio da especificação do teste DF-GLS. Com tais especificações, as quatro primeiras séries da Tabela 2 apresentam como fontes de não-estacionariedade tanto tendência determinista 
quanto estocástica (raiz unitária). Isso porque a hipótese nula de presença de raiz unitária não foi rejeitada pelos dois testes aplicados - as estatísticas de teste são maiores que os valores críticos, para um nível de significância de 5\%. Apenas a taxa de juros com horizonte de previsão de 56 dias não apresenta raiz unitária pelo teste DF-GLS, a 5\% de significância, e também pelo teste Ng-Perron, a $10 \%$.

Considerando os resultados para IR2 e o fato de IR1 ter registrado uma autorregressividade mais elevada, pode-se questionar a presença de raiz unitária em tais séries e comparar o vetor de cointegração (modelo da equação 11) com o modelo tradicional dado pela equação 1 .

O procedimento de análise começou pelo modelo mais geral, especificandose um VAR com três defasagens iniciais. Para manter a comparabilidade entre os critérios de seleção, adotou-se como período amostral da terceira a $42^{\text {a }}$ observação.

Tabela 3. Critérios de informação para seleção de modelo VAR.

\begin{tabular}{cccccc}
\hline Defasagem & LR & FPE & AIC & SC & HQ \\
\hline 0 & NA & 0,000682 & 1,224 & 1,352 & 1,269 \\
1 & 70,216 & 0,000146 & $-0,321$ & $0,191^{*}$ & $-0,137^{*}$ \\
2 & $18,315^{*}$ & $0,000132^{*}$ & $-0,432^{*}$ & 0,464 & $-0,110$ \\
3 & 10,864 & 0,000147 & $-0,345$ & 0,935 & 0,114 \\
\hline
\end{tabular}

* Indica a defasagem selecionada pelo critério,

LR: estatística de teste LR modificada sequencial (Cada teste a 5\% de significância)

FPE: Erro de Predição Final

AIC: Critério de Informação de Akaike

SC: Critério de Informação de Schwarz

HQ: Critério de Informação de Hannan-Quinn

Fonte: Elaboração própria.

Nesse caso, três dos critérios indicaram que a segunda defasagem é a mais adequada para captar a dinâmica do processo tri-variado gerador de dados entre os preços à vista, futuros e taxa de juros, para um horizonte de 28 dias. Um dos requisitos do VAR com duas defasagens é que a especificação seja estável, o que pode ser avaliado por meio das raízes características do polinômio de autorregressivo (ou as inversas do polinômio do operador defasagem) - todas devem estar dentro do círculo unitário.

A Figura 3 mostra que as seis raízes inversas do polinômio de defasagens estão dentro do círculo unitário e que, portanto, a especificação é estável, requisito necessário para a continuidade da análise econométrica. 
Figura 3. Raízes inversas do polinômio característico autorregressivo.

Inverse Roots of AR Characteristic Polynominal

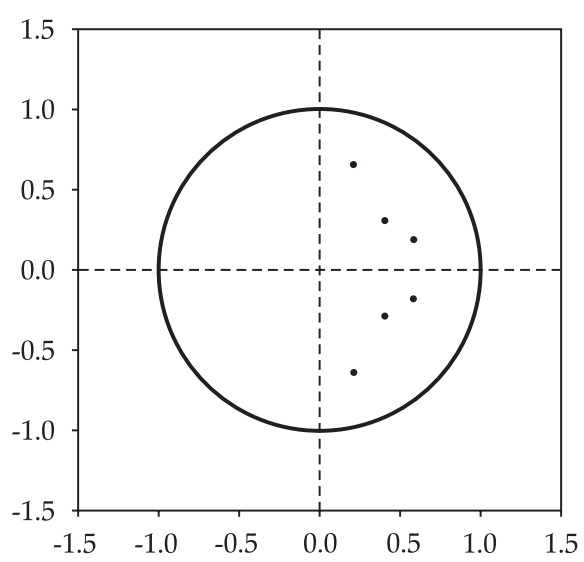

Fonte: Elaboração própria.

Na sequência, avaliou-se o comportamento dos resíduos do VAR quanto à autocorrelação, à normalidade e à heterocedasticidade, a fim de constatar-se quão próximo de ruídos brancos eles estão.

Tabela 4. Testes de Multiplicador de Lagrange para autocorrelação serial.

\begin{tabular}{ccc}
\hline Defasagem & LM- Estat. & p- valor \\
\hline 1 & 12,14 & 0,2054 \\
2 & 10,96 & 0,2786 \\
3 & 6,90 & 0,6472 \\
4 & 8,43 & 0,4906 \\
5 & 8,53 & 0,4815 \\
6 & 1,57 & 0,9966 \\
\hline
\end{tabular}

Hipótese nula: não há correlação serial até a defasagem $\mathrm{h}$

Fonte: Elaboração própria.

A Tabela 4 aponta que não há autocorrelação serial nos resíduos conjuntos do VAR especificado anteriormente, já que as hipóteses nulas são não-rejeitadas a um nível de significância de $5 \%$ (todos os valores de probabilidade - p-valores - são maiores que 5\%, nível de significância escolhido).

Os testes de normalidade conjuntos para o VAR em questão indicaram estatística de Jarque-Bera de 10,48 e respectivo p-valor de 10,56\%, permitindo a não-rejeição da hipótese nula de normalidade multivariada. $\mathrm{O}$ teste para heterocedasticidade 
também resultou na não-rejeição da hipótese nula (homocedasticidade), enquanto o teste conjunto apresentou estatística de 74,47, com respectivo p-valor de $28,1 \%$.

A partir da especificação adequada do VAR, procedeu-se ao teste do traço para a cointegração, com os seguintes resultados para 28 dias de horizonte de previsão:

Tabela 5. Teste do traço para cointegração entre Spot, Fut1 e IR1.

\begin{tabular}{lcccc}
\hline $\begin{array}{c}\text { Hipótese sobre } \\
\text { Número de Vetores } \\
\text { de Cointegração }\end{array}$ & Autovalor & $\begin{array}{c}\text { Estatística } \\
\text { do Traço }\end{array}$ & $\begin{array}{c}\text { Valor Crítico } \\
\mathbf{( 5 \% )}\end{array}$ & p- valor** \\
\hline Nenhum* & 0,459 & 38,11 & 29,79 & 0,0044 \\
No Máximo 1 & 0,249 & 14,12 & 15,59 & 0,0797 \\
No Máximo 2 & 0,073 & 2,97 & 3,84 & 0,0849 \\
\hline
\end{tabular}

* Denote rejeição da hipótese no nível

** p-valores de MacKinnon-Michelis (1999)

Fonte: Elaboração própria.

O teste do traço rejeita a hipótese de nenhum vetor de cointegração, com nível de significância de 5\%, mas não rejeita a hipótese de haver uma relação de longo prazo entre as séries em questão. Assim, inicialmente, há evidências desfavoráveis à eficiência relativa dos mercados de açúcar. O próximo passo é restringir o VAR a um Modelo de Correção de Erro, em que a relação de longo prazo e a dinâmica de curto prazo sejam estimadas em conjunto.

Tabela 6. Vetor de Cointegração (SPOT, FUT1, IR1).

\begin{tabular}{lc}
\hline \multicolumn{1}{c}{ Eq.de Cointegração } & Estimativas \\
\hline SPOT(-1) & 1,000000 \\
FUT1(-1) & $-1,18115$ \\
& $(0,08149)$ \\
& {$[-14,4950]$} \\
IR1(-1) & 0,04538 \\
& {$[0,91579]$} \\
C & 0,03229 \\
\hline Desvios-padrão entre () \& t-estatísticas entre [ ] \\
Fonte: Elaboração própria.
\end{tabular}

O Modelo de Correção de Erro estimado apresenta sinais esperados no vetor de cointegração e insignificância da velocidade de ajustamento para a equação de preços à vista, com estatística $t$ de $-1,50$, bem como da primeira diferença defasada em um e dois períodos. 
Conforme os testes de Wald para exogeneidade e de Granger para causalidade, não há precedência temporal entre as primeiras diferenças dos futuros e a taxa de juros em relação aos preços à vista, o que significa que, no curto prazo, há eficiência.

Nesse ponto, o teste final para confrontar as hipóteses em questão, como já apontado anteriormente, consiste em avaliar se o vetor de cointegração é estatisticamente igual a $[1,-1,1]$. Para tanto, aplica-se o teste de proporção de Lagrange sob a hipótese nula (Tabela 7).

Tabela 7. Teste de Restrição [1, -1, 1] sobre o vetor de cointegração.

\begin{tabular}{lc}
\hline \multicolumn{1}{c}{ Eq. de Cointegração } & Estimativas \\
\hline SPO(-1) & 1,00 \\
FUT1(-1) & $-1,00$ \\
IR1(-1) & 1,00 \\
C & 2,23 \\
\hline Estat. Teste LR & $\mathbf{1 8 , 0 8}$ \\
p-valor & $\mathbf{0 , 0 0 0 1}$ \\
\hline
\end{tabular}

Fonte: Elaboração própria.

O p-valor de $0,01 \%$ ( $<5 \%$, portanto) permite inferir a forte rejeição da hipótese em teste. Ou seja, não é possível afirmar que os parâmetros do vetor de cointegração assumem os valores da hipótese nula, o que implicaria em ineficiência relativa dos mercados. Como a evidência sobre a presença da taxa de juros no vetor de cointegração pode ser questionada, uma vez que, conforme a Tabela 6, esta seria insignificante, realizou-se o teste da hipótese conjunta [1, $-1,0]$. O resultado foi teste LR de 5,934 e p-valor de 5,15\%, não rejeitando a hipótese em teste, arbitrando um nível de significância pouco maior que os 5\% usuais.

A partir desses dados, realizou-se a avaliação do modelo segundo a equação 2, que poderia elucidar a existência de eficiência, com horizonte de previsão de 28 dias. $\mathrm{O}$ mesmo procedimento de modelagem anteriormente descrito foi adotado, sendo um VAR de segunda ordem o melhor modelo ajustado. Os testes para cointegração revelaram a presença de um vetor de cointegração, numa formulação sem tendência linear (Tabela 8).

O teste de restrição sobre esse vetor resultou em teste LR de 4,4 e respectivo valor de probabilidade de 3,59\%, rejeitando a hipótese nula [1, -1]. Nesse caso, ainda que fosse possível discernir entre arbitragem e eficiência, este último teste leva a evidências favoráveis para ineficiência. Em um horizonte de 28 dias de previsão, os preços futuros estão, em média, abaixo dos preços à vista. Assim, para cada ponto percentual de elevação no preço futuro, 28 dias antes do prazo 
de maturidade, espera-se que o preço à vista esteja em média 1,24\% acima dele quando da maturidade do contrato.

Tabela 8. Vetor de Cointegração (SPOT, FUT1).

\begin{tabular}{lc}
\hline \multicolumn{1}{c}{ Eq. de Cointegração } & Estimativas \\
\hline SPOT(-1) & 1,000000 \\
FUT1(-1) & $-1,24090$ \\
& $(0,09342)$ \\
& {$[-13,2825]$} \\
C & 0,03229 \\
\hline
\end{tabular}

Desvios-padrão entre ( ) \& t-estatísticas entre [ ]

Fonte: Elaboração própria.

Para o período de 56 dias, aplicaram-se, novamente, os procedimentos econométricos do geral-para-o-particular, estimando e testando: a defasagem adequada e a estabilidade do VAR; o comportamento conjunto dos resíduos quanto à autocorrelação, heterocedasticidade e normalidade - selecionando-se um VAR com duas defasagens, pela maioria dos critérios de informação, e nãoautocorrelacionado, homocedástico e conjuntamente normal (resultados não reportados por economia de espaço).

O VAR também registrou vetor de cointegração, sem tendência determinista, podendo ser reparametrizado na forma de um modelo de correção de erros. $\mathrm{O}$ teste de restrição sobre os parâmetros do vetor de cointegração, com hipótese nula $[1,-1,1]$, gerou estatística LR de 17,076 com p-valor de 0,0196\%, rejeitando fortemente tal hipótese. Dada a possibilidade de estacionariedade de IR2, já reportada pelos testes de raiz unitária, averiguou-se também a hipótese conjunta $[1,-1,0]$, para qual se obteve estatística LR de 2,093 com p-valor de 35,11\%, não rejeitando as hipóteses em teste.

Na sequência, avaliou-se a cointegração apenas entre SPOT e FUT2, seguindo a mesma metodologia, cujo vetor de cointegração obtido é:

Tabela 9. Vetor de Cointegração (SPOT, FUT2).

\begin{tabular}{lc}
\hline \multicolumn{1}{c}{ Eq.de Cointegração } & Estimativas \\
\hline SPOT(-1) & 1,000000 \\
FUT2(-1) & $-1,12230$ \\
& $(0,0838)$ \\
& {$[-13,3887]$} \\
C & 0,009 \\
\hline
\end{tabular}

Desvios-padrão entre ( ) \& t-estatísticas entre [ ]

Fonte: Elaboração própria. 
Ao impor a restrição [1, -1], obtém-se estatística LR de 1,8179 com p-valor de 17,75\%, não rejeitando tal hipótese. Adicionalmente, os testes de Wald de exogeneidade em bloco (causalidade Granger) evidenciaram a não-rejeição da hipótese nula. Em outras palavras, não há precedência temporal na dinâmica dos preços futuros para o à vista, indicando eficiência de curto prazo. Concluise, portanto, que os mercados são relativamente eficientes num horizonte de previsão de 56 dias.

Tais resultados, porém, precisam ser qualificados. Para o período de 56 dias, metade dos contratos da amostra completa apresenta sobreposiçãoinformacional, já que, nesse horizonte, os vencimentos maio e julho estariam sobrepostos aos de março e maio, respectivamente, interferindo na correspondência amostral.

A segunda, e talvez mais importante, qualificação diz respeito à robustez frente a uma janela amostral que exclua os outliers da taxa de juros - o que, por outro lado, diminui os graus de liberdade e limita a avaliação em termos de comportamento de longo prazo. Ainda assim, procedeu-se a reavaliação dos resultados a partir de 2000, excluindo-se as dez primeiras observações. A Tabela 10 mostra os testes para raiz unitária, suas especificaçoes e valores críticos para a subamostra:

Tabela 10. Testes para raiz unitária - subamostra fev-2000 a set-2007.

\begin{tabular}{lcc}
\hline & DF- GLS & NP \\
\hline Preço à Vista (SPOT) * & $-2,273$ & $-6,945$ \\
Preço Futuro (FUT1) * & $-2,167$ & $-7,32$ \\
Preço Futuro (FUT2) * & $-2,079$ & $-6,494$ \\
Taxa de Juros (IR 1) ** & $-0,293$ & $-14,989$ \\
Taxa de Juros (IR 2) ** & $-0,875$ & $-25,974$ \\
\hline
\end{tabular}

Especificações: * Constante, tendência determinista e nenhuma defasagem (Seleção

MAIC); ${ }^{* *}$ Constante e uma defasagem para IR1 e duas para IR2 (seleção MAIC).

Valores críticos a 5\%: $-3,190$ para DF-GLS e $-17,3$ para NP

Valores críticos a 5\%: -1,951 para DF-GLS e -8,10 para NP

Fonte: Elaboração própria.

Os resultados confirmam a tendência estocástica para as séries de preços. Já para as séries de taxa de juros, há rejeição da hipótese de raiz unitária, com 5\% de nível de significância pelo teste Ng-Perron - considerado mais robusto na presença de estruturas heterocedásticas nos resíduos da equação de teste.

Admitindo-se que as séries de juros sejam estacionárias, a hipótese de eficiência pode ser avaliada por meio da cointegração entre os preços à vista $\mathrm{e}$ futuros, com vetor de cointegração [1,-1]. Para 28 dias de período de previsão, identificou-se um VAR com três defasagens: estável, não-autocorrelacionado e homocedástico, sem componentes deterministas no VAR ou no vetor de 
cointegração, segundo os critérios de informação. O modelo apresentou um vetor de cointegração (rejeitou-se a hipótese de nenhum vetor de cointegração, pelo teste do traço, com estatística de teste de 20,62 e p-valor de $0,16 \%$, e não se rejeitou um vetor, com o de teste de 0,12 e p-valor de 77,22\%). A hipótese nula de restrição sobre o vetor de cointegração foi fortemente rejeitada, com estatística LR de 19,54 e p-valor de 0,001\%.

Para o horizonte de previsão de 56 dias, foram revelados resultados qualitativamente idênticos ao período de 28 dias. Ou seja, a hipótese de eficiência relativa foi rejeitada e, considerando a estacionariedade da taxa de juros e demais componentes do custo de carregamento, o procedimento econométrico de cointegração poderia discernir entre eficiência e arbitragem.

\section{Conclusões}

A hipótese de eficiência de mercado suscita polêmica teórica, bem como requer constante avaliação empírica com aplicações de metodologias cada vez mais robustas, a fim de revelar o grau de conteúdo informacional dos preços formados nos mercados em geral e nos financeiros em especial. No caso deste trabalho, tais evidências auxiliam a tomada de decisão e o posicionamento frente ao risco de agentes que operam nos mercados futuros e à vista de açúcar.

Para tanto, buscou-se responder a seguinte pergunta: a metodologia de cointegração - amplamente aplicada para avaliar a eficiência relativa entre mercados futuros e à vista - seria capaz de discernir entre eficiência e arbitragem nos mercados de açúcar?

A partir do modelo de arbitragem de Brenner e Kroner (1995) e da metodologia de testes comparativos de Kellard (2002), aplicaram-se procedimentos econométricos robustos (cointegração multivariada e modelo de correção de erros), para a avaliação da existência de cointegração entre preços à vista e futuros de açúcar devidamente defasados, em conjunto com a taxa de juros doméstica (como possível elemento não-estacionário do modelo adotado), para os horizontes de previsão de 28 e 56 dias. A hipótese nula do modelo referido é de que essas séries cointegram com coeficientes de $[1,-1,1]$ no vetor de cointegração, o que requer que a taxa de juros defasada, além dos preços, seja diferença-estacionária. A nãorejeição de tal hipótese seria uma evidência favorável ao modelo de arbitragem em detrimento da hipótese de eficiência relativa, uma vez que, se há cointegração entre três séries, não é possível que elas cointegrem duas a duas.

Para o período amostral completo (de junho de 1997 a setembro de 2007), com um horizonte de previsão de 28 dias, obtiveram-se evidências favoráveis à presença de raiz unitária nas três séries, com cointegração entre elas. Porém, não se verificou a hipótese nula do modelo, ou seja, a hipótese de arbitragem não foi aceita. Em contrapartida, testou-se a hipótese de insignificância da taxa de juros defasada no vetor de cointegração, não-rejeitada com valor de probabilidade de 
5,15\%. Em vista desse último resultado, avaliou-se cointegração significante entre os preços, mas não com os coeficientes necessários para evidenciar eficiência relativa. Para o horizonte de previsão de 56 dias, a hipótese de arbitragem foi rejeitada, mas a de eficiência relativa, não, inclusive de curto prazo - o que pode ser explicado pelo fato de, nesse horizonte, haver sobreposição informacional, conforme argumentado anteriormente.

Em busca de robustez, considerou-se que as observações iniciais da taxa de juros para cada horizonte de previsão - muito acima da média do período -, possíveis outliers, pudessem influenciar tanto os testes para a presença de raiz unitária quanto os de cointegração, avaliando-se as evidências para a subamostra de fevereiro de 2000 a setembro de 2007. Nesse caso, mesmo o teste mais robusto para raiz unitária evidenciando a estacionariedade da taxa de juros não garantiu evidências favoráveis à eficiência relativa de longo prazo, em nenhum dos horizontes de previsão.

Em linhas gerais, as evidências empíricas encontradas dão suporte à adequação da metodologia de cointegração para análise de eficiência relativa nos mercados de açúcar, em contraposição à hipótese de arbitragem. Além disso, geram evidências fracas de ineficiência, uma vez que revelam preços futuros defasados sistematicamente inferiores aos preços à vista no longo prazo - ou seja, os futuros seriam previsões viesadas dos preços à vista na data de vencimento dos contratos. No entanto, há que se considerar ainda, à luz das evidências para os dois horizontes de previsão, que tais resultados podem refletir a não-validade de uma hipótese assumida neste estudo, qual seja, a de que o custo de carregamento ( $\mathrm{v}_{\mathrm{t}}$ na equação 7 ) seja estacionário, exceto pelo componente taxa de juros. Em outras palavras, do modelo de arbitragem, considera-se apenas a possibilidade de que a taxa de juros pudesse ser nãoestacionária e o fato de o coeficiente da relação de longo prazo entre preços à vista e futuros estar apontando um viés para baixo pode ser devido à omissão de algum outro fator não-estacionário na relação de longo prazo - o custo de estocagem e/ou o retorno conveniente esperado pelo investidor, ambos considerados estacionários.

Abre-se, portanto, espaço para a realização de pesquisas futuras que busquem identificar proxies adequadas para essas variáveis (custo de estocagem e retorno conveniente), a fim de reavaliar a adequação do modelo de arbitragem frente à eficiência relativa, tanto para o mercado de açúcar quanto para outras importantes commodities brasileiras.

\section{Referências Bibliográficas}

BRENNER, R. J.; KRONER, K. F. Arbitrage, Cointegration, and Testing the Unbiasedness Hypothesis in Financial Markets. The Journal of Financial and Quantitative Analysis, Vol. 30, No. 1, p. 23 - 42, Mar - 1995. 
Mercados Futuro e à Vista de Açúcar:

uma análise empírica de eficiência versus arbitragem

CAMPBELL, J. Y.; ANDREW W. Lo.; MACKINLAY A. C. The Econometrics of Financial Markets. Princeton University Press, 1997.

CARGILL, T. F; RAUSSER, G. C. Temporal Price Behaviour in Commodity Futures Markets. Journal of Finance, v. 30, n. 4, p. 1043-1053, sep. 1975.

COCHRANE, J. H. A Critique of the Application of Unit Root Tests. Journal of Economic Dynamics and Control, 15, p. 275-384, 1991.

DeJONG, D.N.; NANKERVIS, J.C.; SAVIN, N.E.; WHITEMAN, C.H. The Power Problems of Unit Root Tests for Time Series with Autoregressive Errors. Journal of Econometrics, 53, 323-342, 1992.

DICKEY, D.; FULLER, W. A. Likelihood Ratio Statistics for Autoregressive Time Series With a Unit Root. Econometrica, v. 49, n. 4, p. 1057-72. jul. 1981.

ELLIOTT, G., ROTHENBERG, T.J., STOCK, J.H., Efficient tests for an autoregressive unit root. Econométrica, 64, 813-836, 1996.

ENDERS, W. Applied Econometric Time Series. New York: John Wiley \& Sons, 2004.

FAMA, E. Efficient Capital Markets: A Review of Theory and Empirical Work. Journal of Finance, v. 25, p. 383-417, may 1970.

FAMA, E.; FRENCH, K. R. Commodity Futures Prices: Some Evidence on Forecast Power, Premiums, and the Theory of Storage. Chicago: The University of Chicago Press. Journal of Business, v. 60, n. 1, p. 55-73, jan. 1965.

FAMA, E. Random Walks in Stock Market Prices. Universiy of Chicago, paper. n.16, p. 55-59, september. 1965.

HULL, J. Options, Futures, and Other Derivatives. Prentice-Hall Inc., 1997.

JOHANSEN, Soren. Estimation and Hypothesis Testing of Cointegrated Vectors in Gaussian Vector Autoregressions. Econometrica, v. 59, p. 1551-80, nov. 1991.

JUSELIUS, K. The Cointegrated VAR Model: Methodology and Applications. New York: Oxford University Press, 2006. 457p.

KELLARD, N.; NEWBOLD, P.; RAYNER, T.; ENNEW, C. The Relative Efficiency of Commodity Futures Markets. The Journal of Futures Markets, Vol. 19, No. 4, pp. 413-432, 1999.

KELLARD, N. Evaluating Commodity Market Efficiency: Are Cointegration Tests Appropriate? Journal of Agricultural Economics, Vol. 53, No. 3, pp. 513-529, 2002.

LARSON, A. B. Measurement of a Random Process in Future Prices. Food Research Institute Studies, v. 1, n. 3, nov. 1960. 
LIMA, R. C.; OHASHI, A. The Efficient Market Hypothesis and the Dynamic Behavior of Sugar Future Prices. Departamento de Economia/PIMES - Universidade Federal de Pernambuco, 1999.

MADDALA, G.S.; KIM, I.M. Unit Roots, Cointegration and Structural Change. Cambridge University Press, Cambridge, 2003.

MALKIEL, B. G. The Efficient Market Hypothesis and Its Critics. Journal of Economic Perspectives, v. 17, n. 1, p. 59-82, winter, 2003.

McKENZIE, A. M.; JIANG, B.; DJUNAIDI, H.; HOFFMAN, L. A.; WAILES, E. Unbiasedness and Market Efficiency Tests of the U.S. Rice Futures Market. Review of Agricultural Economics, Vol. 24, No. 2, pp. 474-493, 2002.

NG, S., PERRON, P. Useful Modifications to Some Finite Sample Distributions Associated with a First-order Stochastic Difference Equation. Econometrica, 45, 463-485, 1996.

NG, S., PERRON, P., Lag length selection and the construction of unit root tests with good size and power. Econometrica, 69, 1519-1554, 2001.

PESARAN. M. H. Market Efficiency Today. Institute of Economic Policy Research. University of Southern Califórnia, dec. 2005.

STEVENSON. R. A.; BEAR. R. M. Commodity Futures: Trends or Random Walks? Journal of Finance, v. 25, n. 1, p. 65-81, mar. 1970.

WANG, H. H. Is China's Agricultural Futures Market Efficient? 25th International Conference of Agricultural Economists, Durban, South Africa, August 16-22, 2003. 
